\title{
AC 2011-1962: CONCEPT INVENTORY ASSESSMENT INSTRUMENTS FOR CIRCUITS COURSES
}

\author{
Tokunbo Ogunfunmi, Santa Clara University
}

TOKUNBO OGUNFUNMI, Ph.D., P.E. is the Associate Dean for Research and Faculty Development in the School of Engineering at Santa Clara University, Santa Clara, California. He is also an Associate Professor of Electrical Engineering and Director of the Signal Processing Research Lab. (SPRL).

He earned his BSEE (First Class Honors) from Obafemi Awolowo University (formerly University of Ife), Nigeria, his MSEE and PhDEE from Stanford University, Stanford, California. His teaching and research interests span the areas of Circuits and Systems, Digital Signal Processing (theory, applications and implementations), Adaptive Systems, VLSI/ASIC Design and Multimedia Signal Processing. He is a Senior Member of the IEEE, Member of Sigma Xi, AAAS and ASEE.

\section{Mahmudur Rahman, Santa Clara University}

Dr. Mahmudur Rahman received M.S. Engg. and Dr. Engg. from Tokyo Institute of Technology, and then worked as a research scientist in NEC Corporation at Tamagawa, Tokyo, Japan during 1981 -1985. He actively co-organized 1st through 5th International Conference on Silicon Carbide and Related Materials in various capacities including Conference Chair and Editor of Conference Proceedings during 1987-1993. Presently he is an Associate Professor of Electrical Engineering and Director of the Electron Devices Laboratory at Santa Clara University. His current research interests include: i) Stable High Current Density Carbon Nanotube Cold Field Emission Electron Source Technology ii) Wavelet Approach to Systematic Quantitative Characterization of Surface Nanostructures of Thin-Films based on Scanning Probe Microscopy, iv) Statistical and Temperature Map Dependent Electromigration Modeling and Simulation of Deep-Submicron Processes Applied to Design for Manufacturing, v) Low Cost Efficient Thin-film Organic Solar Cells by Optimization of Pentacene/Fullerene Interface.

He was the chair of the IEEE Electron Devices Society, Santa Clara Valley Chapter, in 1989. He is a senior member of the IEEE. 


\title{
Concept Inventory Assessment Instruments for Circuits Courses
}

\begin{abstract}
Electric Circuits course is a core component of undergraduate curricula in electrical engineering programs worldwide.

The Electric Circuits Concept Inventory (ECCI) is a set of multiple-choice questions that we are developing to measure students' understanding of fundamental concepts such as DC and AC Circuits. First and Second-order circuits, etc. and Advanced Circuit analysis topics are covered. These questions do not test problem solving steps but test major concepts and ability of students to understand the concepts in the context of the problem and apply the required methods to solve the problems.
\end{abstract}

We plan to collect and collate all similar concepts questions (generated by others and ourselves) and build up an ECCI test database that can be used in every offering of Circuits course at all universities who wish to participate. We also encourage other faculty to adopt the test. This test fulfills the United States Accreditation Board for Engineering and Technology (ABET) requirement for assessment. It also helps track the effectiveness of teaching styles by testing whether the students are learning the basic concepts in the course.

In this paper, we first review some assessment instruments used previously in the Circuits courses. We classify the major ideas and concepts that need to be tested. Then we present several examples of possible multiple-choice questions designed to test fundamental concepts which students are expected to learn in the Circuits courses.

We examine the various concepts tested in each question and relate its importance to a quality overall course in Electric Circuits. We also report on the outcomes of these new assessment instruments at the conference. 


\section{Introduction}

Electric circuits are a part of the basic fabric of modern technology. The use of electrical and electronic devices and systems has become essential in all aspects of a modern society as such, all engineering majors require a first course in electrical circuits in their core curriculum. This course is intended to serve the purpose of educating an engineering student about the fundamental behavior of the five basic individual active and passive elements, and further the basic concepts and laws that govern their group behavior when these individual elements are connected in a circuit or a system. It is important to note that while basic electrical elements obey certain group behavior when they are connected in a circuit or system, they do so maintaining their individual properties intact. From their direct experience in teaching the basic circuits course to multidisciplinary engineering classes over a most recent five-year period, the authors have been able to identify conceptual situations the students find it most difficult to handle. This paper contains examples resulting from their efforts in designing and implementing their circuit courses effectively with a goal to clarify the conceptual contents.

The ECCI is a set of multiple-choice questions that measures students' understanding of DC Circuit analysis. The topics include (i) fundamental conceptual circuit topics such as the Relationship between Potential (Volt), Charge (Coulomb) and Energy (Joule), Polarity of voltage sources, Validity of a circuit, Independent and Dependent current/voltage sources, Short and Open circuit conditions, Resistors in Series/Parallel and Y- $\Delta$, behavior of DC voltages in Capacitors and DC currents in Inductors ; (ii) Circuit laws such as Ohm's law, Kirchhoff's Law, and circuit theorems such as Linearity and Superposition, Source Transformation, Thevenin's and Norton's Equivalence ; and (iii) Circuit analysis techniques such as Nodal and Mesh analysis.

In Section II, the pedagogical approach adopted by the authors is enumerated. The rationale behind a conceptual inventory is discussed in Section III followed by major topics in a DC circuit course listed in Section IV. The concept inventory of interest is presented in Section V in the form of examples testing the fundamental conceptual circuits topics. For each example, the concept involved is first identified and explained, followed by a circuit diagram and a question. Projected applications of the concept inventory are explored in Section VI before concluding the paper in Section VII.

\section{A Novel Pedagogical Approach}

A concept (abstract term: conception) is a cognitive unit of meaning — an abstract idea or a mental symbol sometimes defined as a "unit of knowledge," built from other units which act as a concept's characteristics. A concept is typically associated with a corresponding representation in a language or zymology such as a single meaning of a term.

The theoretical background of linear circuit theory is derived from Maxwell's theory of electromagnetism. The applications of circuit theory is essentially based on the key concepts of Kirchoff's Laws, impedance, Ohms Law and the Principle of Superposition ${ }^{1-3}$. Using these concepts any linear circuit can be solved under a given set of conditions. One of the most 
interesting concepts that arise from the linear circuit theory is the equivalent circuit such as Thevenin's equivalent circuit and Norton's equivalent circuit ${ }^{4-6}$.

A course in circuit analysis is perhaps the first exposure students have to electrical engineering. In teaching this course it is important that one avoids wordiness and giving details that may hide concepts and impede understanding of the material.

Instead, problem solving should be approached as a thought process in which one applies conceptual understanding to the solution of a practical problem which involves an effort to build an understanding of concepts and ideas explicitly in terms of previous learning.

The learning challenges faced by students of electric circuits are prodigious; each new concept is built on a foundation of many other concepts where the students are encouraged to think through problems based on concepts and not only follow a set of problem solving procedure usually outlined in text books. Every new problem is an interwoven conceptual hurdle.

Our goal is to help students recognize how new concepts or ideas fit together with those previously learned. Computer tools such as MATLAB, EXCEL, and PSPICE cannot replace the traditional methods for mastering circuits.

An appropriate pedagogical approach using a set of teaching principles should consider the aspects that:

1. Circuit analysis can be fun

2. It is a marvelous education in logical thinking

3. Application aspects of concepts covered toward a design

4. Concepts applied in real life situations

5. Learning from mistakes and reminders not to make some particular mistakes

6. Checking solutions using concepts

\section{Concept Inventories}

The idea of a concept inventory was initially presented by David Hestens et al. in The Physics Teacher for a course in Physics on Force ${ }^{7}$. Since then there have been several attempts to use the idea in several aspects of electrical engineering such as electromagnetics, signals and systems, thermodynamics, etc. ${ }^{8-10}$

For example, the Signals and Systems Concept Inventory (SSCI ${ }^{8}$ is a set of multiple-choice questions that measures students' understanding of fundamental concepts such as signal transformations, linearity, time-invariance, transforms, convolution, etc. There are two versions of the SSCI for Signals and Systems. One deals with Continuous-Time (CT) signals and systems and the other deals with Discrete-Time (DT) signals and systems. The SSCI has been administered in several universities as a component of student learning assessment that is required by the American Board for Engineering and Technology (ABET) Engineering Accreditation Commission to ensure student learning ${ }^{11-12}$.

A literature search revealed that there was some effort to produce a Concept Inventory questions for an Electric Circuits course in the past. We were unable to get the original authors to respond 
to our enquiries about their previous work on the subject ${ }^{24-27}$. Also, the ECCI is different from the EIT or the PE exams that are needed for professional engineers registration. The ECCI is designed to test conceptual understanding and presents a different pedagogical approach. In addition, the EIT and PE exams include other materials not just on electric circuits.

Regardless of previous work, we believe the approach we have taken here is pedagogically sound and leads to questions that test concepts and make help student learning.

There are many textbooks in use for teaching electric circuits courses ${ }^{13-19}$. Many of these textbooks present the key concepts, however in our experience students find some of these concepts difficult to grasp.

In teaching Electric Circuits courses, these key concepts are important and need to be tested so as to ensure student learning and comprehension. We have developed some multiple-choice questions suitable for testing some of these concepts ${ }^{20-21}$. These questions are somewhat different from some of the online interactive ones in the overall pedagogical approach and conceptual contents ${ }^{22-23}$. We invite other academics/researchers in this area to make available their concept questions as we develop a universal database of such questions that can be used for assessing student learning of key concepts.

IV Major Topics in DC Electric Circuits Course

The major topics taught in a DC Circuit course can be summarized into three parts as follows:

Introduction to DC Circuits:

- Concept of Charge, Current and Voltage

- Concept of Power and Energy

- Introduction to Circuit Elements

- Ohm's Law, Nodes, Branches and Loops

- Kirchhoff's Laws, Voltage/Current Division

- Wye-Delta Transformations

DC Circuit Analysis Methods

- Nodal and Mesh Analysis

- Circuit Linearity Properties

- Source Transformations

- Norton's Theorem

- Thevenin's Theorem

- Maximum Power Transfer

Other DC Circuit Analysis Methods

- Operational Amplifiers

- Capacitors and Inductors

- First-Order Circuits

- Second-Order Circuits 
These topics are the basic minimum covered in a typical first course on DC Circuits. A second course may focus on AC Circuit Analysis and cover other topics such as Sinusoidal Steady-state analysis, AC Power Analysis, Three-Phase Circuits, magnetically Coupled Circuits, Two-port networks, etc.

\section{Concept Inventory of Examples}

In this section a number of key concepts are presented along with circuits used to explain its application. The conceptual topic is first identified and symbols or notations used clearly explained followed by an example question in reference to a circuit. Common misconceptions and difficulties students experienced in classes the authors taught as collected from student homework, quizzes, and examinations are also discussed.

\section{V.1 Fundamental Conceptual topics}

In this subsection examples which deals with fundamental concepts associated with basic circuit elements and their different configurations are presented .

1) concept: Relationship between Potential (Volt), Charge (Coulomb), and Energy (Joule)

This example illustrates the relationship between three most fundamental electrical quantities that Energy or Work (Joule) $=$ Charge (Coulomb) x Potential (Volt). It introduces the concept of a "reference potential" which is $0 \mathrm{~V}$ for the case when the charges are at infinity, and that the Potential of any other point in space acquires a polarity depending on whether negative or positive Charges are moved from infinity to that a point.

Students who are introduced to these quantities for the first time are mostly confused about the polarities of the Potential and under what condition work is done on the charges as against by the charges.

Q. Potential difference from point $\mathrm{C}$ to $\mathrm{F}$ is $1.65 \mathrm{~V}$. It takes $2.56 \mathrm{~nJ}$ to move $6 \times 10^{9}$ protons from A to $\mathrm{C}$. How much work needs to be done to move $3 \times 10^{8}$ electrons from F to $\mathrm{A}$ ?

2) concept: Polarity of voltage sources

In this example the conventional polarity notation scheme in voltage sources is clarified. In a circuit diagram, terminals of voltage sources denoted by + or - does not necessarily indicate that it is at positive or negative potential, rather the terminal denoted by + is at a higher potential than that denoted by - . Both terminals can be in reality at positive, negative or a combination of both polarities of potential. The magnitude of potential difference between the potential of the terminal marked higher $\left(V_{H}\right)$ and that marked lower $\left(V_{L}\right)$, i.e., $V_{H}-V_{L}$, 
is shown next to the voltage source symbol, sometimes calling it a voltage rise, for example, when writing a loop equation.

The authors noted that this notation scheme is highly confusing to a student as they tend to get stuck with the notion that that a terminal marked + is invariably at a positive Potential and that marked -- at a negative Potential. The authors found it to be useful not to use + or for such purpose, rather use the symbols $\mathrm{V}_{\mathrm{H}}$ for higher and $\mathrm{V}_{\mathrm{L}}$ for lower potential of the voltage source terminals.

Q. Find the voltages $V_{1}$ and $V_{2}$ as indicated in Figure 1 .
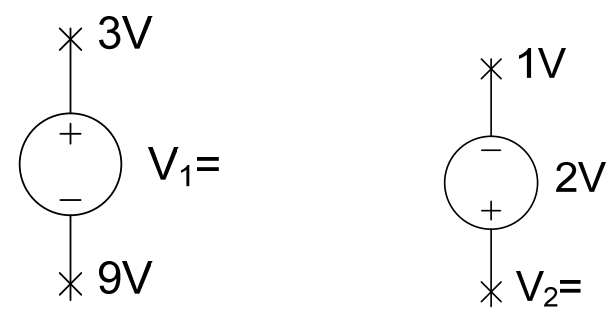

Figure 1. Finding Polarity of Voltage Sources

\section{3) concept: Uniqueness of node voltages and branch currents in a real circuit}

This example illustrates the concept that a point in a circuit has a unique polarity and a branch can only flow a unique current.

Students tend to ignore this important reality and end up with denoting a node having more than one potential and more than one current flowing in a branch depending on other circuit element. This tendency creates serious mistakes when student try to write node and loop equations using imaginary loop currents and node voltages.

Q. Determine whether the circuits are valid or not and why.
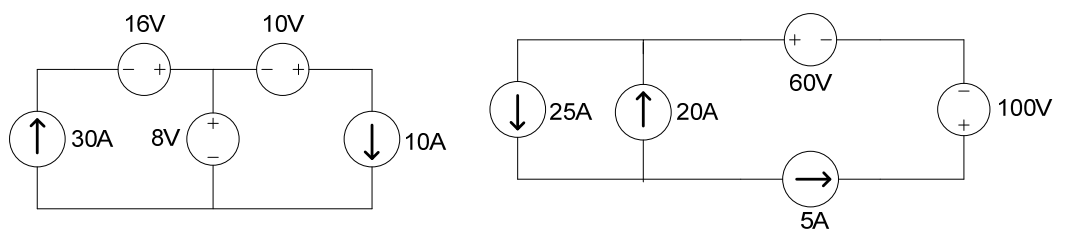

Figure 2. Finding Validity of a Circuit

4) concept: In a valid circuit power delivered = power absorbed

The concept of under what conditions power is being delivered or absorbed can be unambiguously expressed in terms of the direction of current through or voltage across circuit elements in a circuit. Using these conceptual notations, the validity of a circuit can be determined.

This is a very useful concept which can be used readily by the students to verify whether 
their solution of the circuit is indeed correct. The use of this verification builds up their confidence in circuit solutions all by themselves.

The average students in classes tend to rely on solution manuals or on someone else who will give them solutions. Once the students have access to a solution, it allures them away from the motivation to think and consequently learning and using concepts takes a back stage. Using of this concept by students make them self sufficient.

Q. Calculate power delivered and power absorbed by the elements in the circuit.

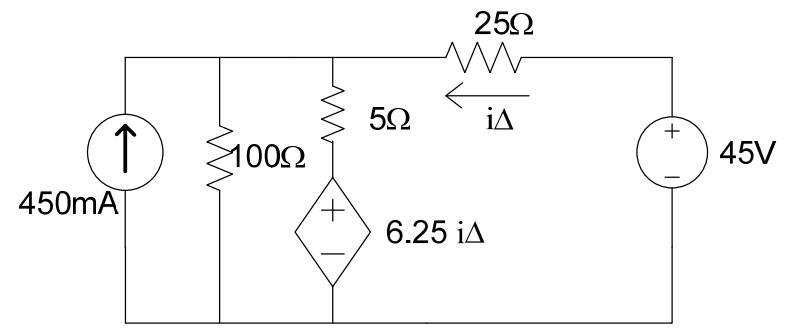

Figure 3. Finding Validity of a Circuit

5) concept: Independent and Dependent current/voltage sources.

The concepts that an independent voltage or current source can allow any amount of current through it or any voltage across its terminals, respectively, is clarified in this example. The relationship between the current and the voltage in a dependent current or voltage source can either be a constant, a resistance, or a conductance. A circuit containing only dependent sources is not an active circuit.

Students take a while to be familiar with the situation that the current and voltage in a circuit can be inter-dependable.

Q. Sketch and Label the I-V relationship for the following current/voltage sources.

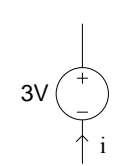

( a)

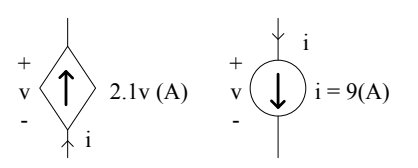

(c)

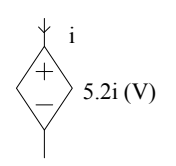

(b)

(d) 
Fig. 4. Independent and Dependent current/voltage sources.

\section{6) concept: Resistors in Series/Parallel}

In a circuit, several resistances can be connected so that they carry the same current (a series connection), or they can be connected such that each of them have the same potential across it (parallel connection), or a combination of both.

This example illustrates the concept of equivalent resistance across any two terminal points which can be calculated by considering the circuit to be a combination of series and parallel-connected resistors.

The students find it difficult to identify which elements in a given circuit are in series and which are in parallel. Redrawing the given circuit, such as shown in this example, by identifying each node with a specific name and verifying the nodes which have the same voltages or branches which have the same current help identify which are in series and which are in parallel.

Q. Find the equivalent resistance $\mathrm{R}_{\mathrm{ab}}$ in the circuits given.

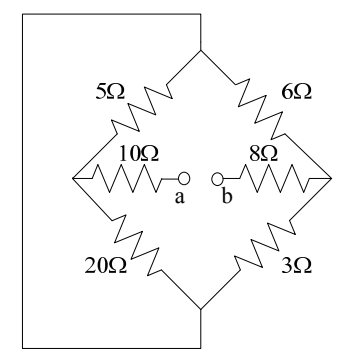

Figure 5. Finding Equivalent Resistors Across Two Terminals

\section{7) concept: Short and Open circuit}

The concepts of a short circuit when the potential across a pair of terminals is $0 \mathrm{~V}$, and an open circuit when the current flowing along a branch is $0 \mathrm{~A}$ is very important in circuit situations and is illustrated in the following example. Open circuit situation occurs when a branch is not a part of a closed loop, and a short circuit often occurs when there is no resistance between the two terminals of interest. Consequently, there is no potential drop along a resistive open circuit branch.

Students often ignores these situations which when considered can minimize the work needed to solve a circuit. 
Q. Find $V_{1}, V_{2}, V_{3}, I_{1}$, and $I_{2}$, in the circuit $s$ below
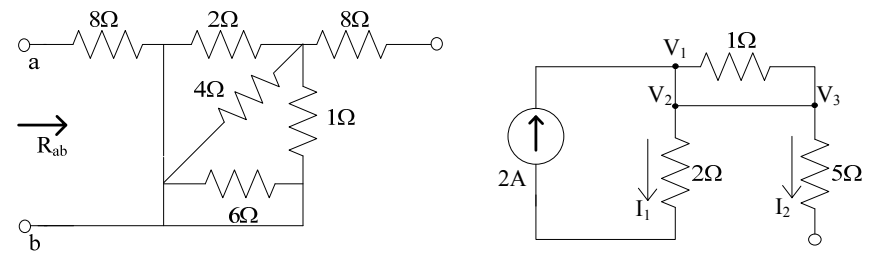

Figure 6. Testing Concepts of Short and Open Circuit

8) concept: Potential (current) cannot change instantaneously in a capacitor (inductor).

The concept that it takes an infinite amount of current (voltage) to bring about a change in the dc voltage (current) across a capacitor (inductor) instantaneously, consequently the voltage (current) across (along) a capacitor (an inductor) cannot change instantaneously is illustrated in this example shown in Figures 7 and 8.

Students find it difficult to realize that unlike in circuits containing only resistors, in circuits having circuit elements such as capacitors and inductors, the current and voltages are not at steady state but time dependent, requiring a transient analysis of the circuit even if all active sources are dc.

Q. Sketch and Label the current/voltage response for the given current/voltage inputs in the following circuit elements.

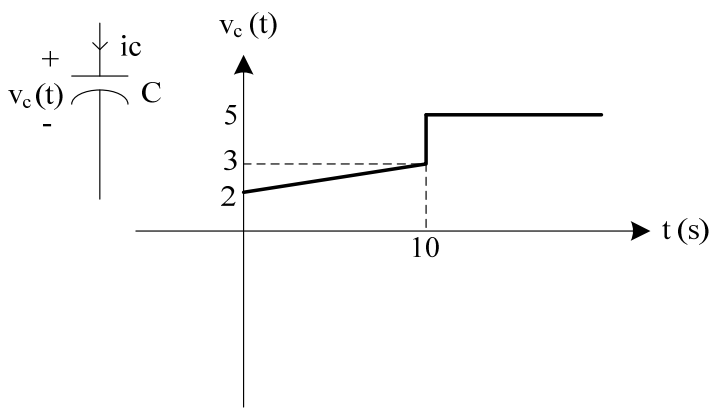

Figure 7. Instantaneous change in Voltage in an Inductor

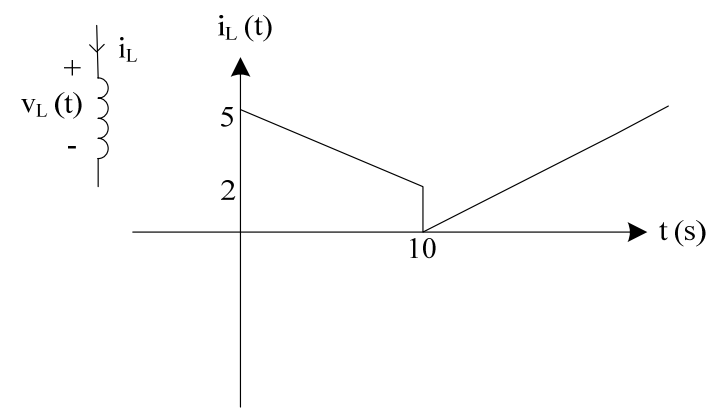

Figure 8. Instantaneous change in Current across a Capacitor

\section{2 DC Circuit laws and theorems}

In this subsection examples explaining circuit laws such as Ohm's Law, Kirchoff's Laws, and circuit theorems such as Linearity and Superposition, Source Transformation, Thevenin's and Norton's Equivalence are presented.

1) concept: Resistors in Wye/Delta

The Wye and Delta configurations of connecting resistors in a dc circuit are very useful 
to investigate because of implication in 3-phase AC circuits. The resistor values connected in Wye can be interchanged with equivalent resistor values when they are connected in Delta configuration. This example illustrates how the input resistance across any arbitrary pair of terminals can be determined by multiple Delta-Wye conversions.

Q. Determine the input resistance $\mathrm{R}_{\text {in }}$ in the network given in Figure 11 by using Delta-Wye conversions.

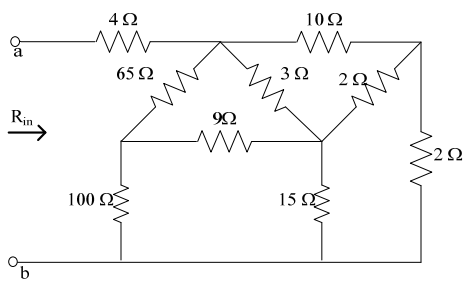

Figure 9. Input Resistance using Wye-Delta conversions

2) concept: Thevenin's equivalent circuits: Different solution approaches for two different types of circuits:

The concept that a circuit having only dependent sources cannot have a Thevenin's equivalent open circuit voltage and that the Thevenin's equivalent resistance in such case is extracted by applying an external voltage source is illustrated in the examples shown in Figure 10. The circuit in Figure 11, however, has a $3 \mathrm{~mA}$ independent current source.

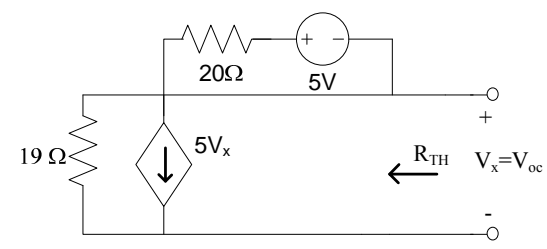

Figure 10. Finding Thevenin's Equivalent circuit for a circuit which does not have independent active sources

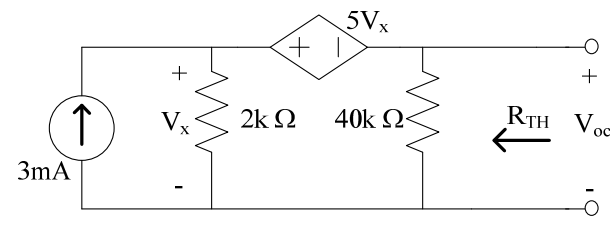

Figure 11. Finding Thevenin's Equivalent for a circuit with independent active sources

3) concept: A resistance in series (parallel) with a current (voltage) source has no effect on the equivalent circuit w.r.t. two arbitrary terminals.

This example illustrates the special situation when a resistor connected in series with an 
independent current source and a resistance connected across an independent voltage source can be ignored for solving the circuit across any two terminals. This is the concept of a sub circuit illustrated further in the next example shown in Figure 12. In this circuit, the $16 \Omega$ resistor and the $260 \Omega$ resistor do not affect the output voltage $\mathrm{V}_{\mathrm{o}}$.

Q. Use source transformation to find $\mathrm{V}_{\mathrm{o}}$ in the circuit.

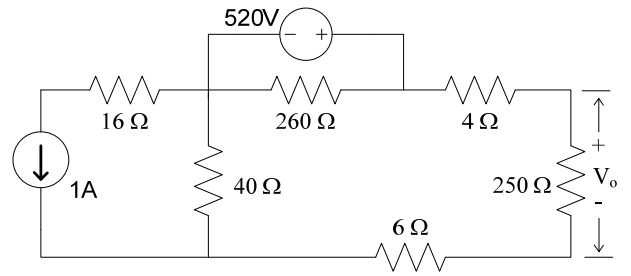

Figure 12. Resistance in series (parallel) with a current (voltage) source

4) concept: Sub-circuits connected across same independent voltage source do not influence each other.

When several circuit elements are connected across an independent voltage source, a sub circuit is created. The example in Figure 13 shows how sub circuits are independent of each other. Consequently, the $6 \Omega-9 \Omega-1 \mathrm{~mA}$ sub circuit does not affect the current and voltages in elements right of the $2 \mathrm{~V}$ voltage source.

Q. Convert the circuit left of terminals a-b to a single current source in parallel with a single resistor using source transformation.

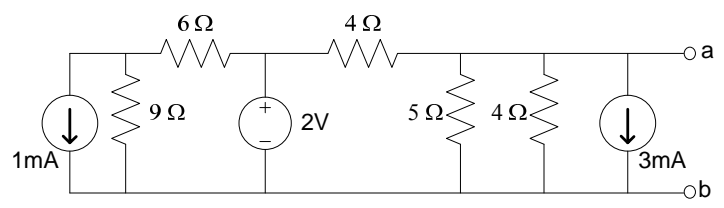

Figure 13. Sub-Circuits connected across same independent voltage source.

\section{3 DC Circuit analysis techniques}

In this subsection examples illustrating some specific issues for solving circuits using Mesh and Nodal analysis are presented.

1) concept: current (voltage) source in a mesh (node) equation.

In mesh (nodal) analysis, voltages across elements in a closed mesh (currents entering and exiting a node) is sought. Students often are confused when they come across current (voltage) sources in a mesh (at a node) which is clarified in the following examples in Figures 14 and 15. 
When attempting to solve Figure 14 using Mesh Equation, it is necessary to assume a voltage across the dependent current source $3 \mathrm{I}_{0}$. The number of total unknowns remains the same by doing so. Similarly, it is necessary to assume a current through the $10 \mathrm{~V}$ source to solve circuit in Figure 15.

Q. Write appropriate mesh (node) equations to solve for $\mathrm{V}_{\mathrm{o}}\left(\mathrm{I}_{\mathrm{o}}\right)$ in the circuits below.

i. use mesh equations only

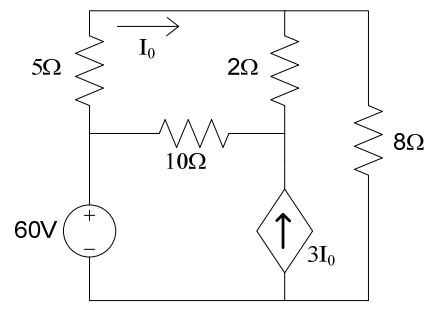

Figure 14. Current Source in a Mesh Equation

ii. use node equations only

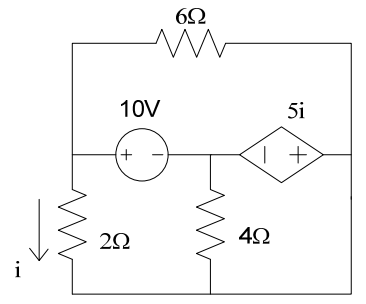

Figure 15. Voltage Source in a Node Equation

2) concept: Disruption of circuit by insertion of voltmeter/ammeter.

Since an ammeter or a voltmeter has very small or very large resistance, respectively, insertion of these devices in a given circuit to measure current and voltage essentially changes the circuit and the measured values are different than those in the original circuit. This situation is explained through this example shown in Figure 8. 
Q. A voltmeter is used to measure $\mathrm{V}_{\mathrm{o}}$ in the circuit. Calculate percentage error introduced by the voltmeter.

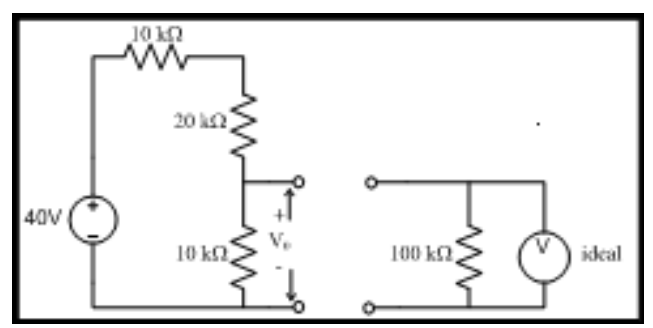

Figure 16. Circuit disruption by insertion of Voltmeter/Ammeter

\section{Applications and Initial Assessment Results}

The examples presented in this paper in Section $\mathrm{V}$ have been taken from examinations over a period of three years. During each year, difficulties, common errors, and misconceptions students experienced for a certain conceptual circuit were investigated and documented. In each subsequent year, a particular concept was presented to the class based on the finding in a previous year by addressing the issues. The authors assessed the improvements in the presentation of the concepts from exam grades obtained by students and found that application of this concept inventory in their pedagogical approach to enhance the quality of the understanding.

Although it is not a rigorous statistical study, a consistent trend has been observed in scores of the quizzes and midterm examination of three classes taught by co-author Rahmanin spring of 2007 (31 students), 2008 (35 students) and 2009 (44 students) in ELEN 50, the first course in circuits where the concepts discussed in this paper were introduced. Students' mistakes in conceptual questions in quizzes were corrected and discussed individually as a feed back to each student. The average scores of quizzes when the concepts are first introduced have been 10-20\% lower in each year compared those in the midterms when the same concepts were introduced again after discussing and correcting students mistakes and misconceptions made in the quizzes.

Also an increasing trend in the enrollment in successive years was observed indicating increased positive perception among the student body about the methodology used in presenting the material in these classes as evidenced in written student statements on evaluation of these three courses.

\section{Conclusions}

In this paper, we presented a set of multiple-choice questions suggested for inclusion in the ECCI focusing on DC Circuit laws and DC Circuit analysis techniques. These questions do not 
test problem solving steps but test major concepts and ability of students to understand the problem and apply the required methods to solve the problem. We examine the various concepts tested in each question and relate its importance to a quality overall course in Electric Circuits. We welcome more additions to this growing repertoire of questions that we are building to use in finalizing the ECCI test especially in DC and AC Circuit analysis topics.

Possible goals of the use of such ECCI test questions include the following: (1) To determine how much conceptual understanding the students have developed by the end of the course (compared to the beginning of the course). (2) To correlate the performance on the end-of-term exam with the performance on the ECCI Post-Test which is designed to test both the conceptual understanding and the steps of problem solving and system design. (3) To determine the concepts which the students have had difficulty understanding so that there may be more emphasis on those concepts the next time the course is offered. (4) To relate the performance of students in Electric Circuits course material (ECCI) with the other Concept Inventories such as SSCI for Signals and Systems courses.

\section{References}

[1] Origins of the Equivalent Circuit Concept, Don H. Johnson, Rice University, August 11, 2001.

[2] G.S. Ohm. Die galvanische Kette, mathematisch bearbeitet [A mathematical theory of Galvanic circuits]. 1827.

[3] H. Helmholtz. II. Uber einige Gesetze der Vertheilung elektrischer Str"ome in k"orperlichen Leitern mit Anwendung auf die thierisch-elektrischen Versuche [Some laws concerning the distribution of electrical currents in conductors with applications to experiments on animal electricity]. Annalen der Physik und Chemie, 89(6):211-233, 1853.

[4] L. Th'evenin. Extension de la loi d'Ohm aux circuits 'electromoteurs complexes [Extension Of Ohm's law to complex electromotive circuits]. Annales T'el'egraphiques, 10:222-224, 1883. .

[5] L. Th'evenin. Sur un nouveau th'eor'eme d'electricit'e dynamique [On a new theorem of Dynamic electricity]. C. R. des S'eances de l'Acad'emie des Sciences, 97:159-161, 1883.

[6] E.L. Norton. Design of finite networks for uniformfrequency characteristic. Technical Report TM26-0-1860, Bell Laboratories, 1926.

[7] David Hestenes, Malcolm Wells and Greg Swackhamer, "Force Concept Inventory", The Physiscs Teacher, vol. 30 (3), pp. 141-151, 1992.

[8] Kathleen Wage et. al. "Signal and Systems Concept Inventory", IEEE Transactions on Education, vol. 48, No. 3, pp. 448-461, August 2005

[9] Branislav M. Notaros, "Concept Inventory Assessment Instruments for Electromagnetics Education", Proceedings of the IEEE International Symposium on Antennas and Propagation, 2002.

[10] D. L. Evans and David Hestenes, "The Concept of the Concept Inventory Assessment Instrument", Proceedings of the Frontiers in Education Conference, Reno, Nevada, Oct. 10-13, 2001.

[11] American Board for Engineering and Technology (ABET) Engineering Accreditation Commission, Criteria for Accrediting Engineering Programs, Baltimore, MD, 2003. (URL: http://www.abet.org ) 
[12] Felder et. al., Index of Learning Styles, papers, (URL: http://www.ncsu.edu/felder-public/ILSpage.html)

[13] William H. Hayt, Jr., Jack E. Kemmerly, and Steven M. Durbin, Engineering Circuit Analysis, 7th ed., McGraw Hill, New York, 2007.

[14] Charles K. Alexander and Matthew N. O. Sadiku, Fundamentals of Electric Circuits, 4th ed., McGraw Hill, New York, 2009.

[15] Ronald E. Thomas, Albert J. Rosa, and Gregory J. Toussaint, The Analysis and Design of Linear Circuits, 6th ed., John Wiley, Hoboken, NJ, 2009.

[16] Allan R. Hambley, Electrical Engineering, Principles and Applications, 4th ed., Prentice Hall, Upper Saddle River, NJ, 2008.

[17] J. David Irwin, and R. Mark Nelims, Basic Engineering Circuit Analysis, $9^{\text {th }}$ ed., John Wiley, New York, 2008.

[18] Richard C. Dorf, and James A. Svoboda, Introduction to Electric Circuits, 7th ed., John Wiley, Hoboken, NJ, 2006.

[19] James W. Nilsson and Susan A. Riedel, Electric Circuits, 7th ed., Prentice Hall, Upper Saddle River, NJ, 2005.

[20] Tokunbo Ogunfunmi and Mahmud Rahman, "A Concept Inventory for an Electric Circuits Course: Rationale and Fundamental Topics", Proceedings of the 2010 IEEE ISCAS Conference.

[21] Mahmud Rahman and Tokunbo Ogunfunmi, "A Set of Questions for a DC Circuits Course”, Proceedings of the 2010 IEEE ISCAS Conference.

[22] The University of Texas, Online Circuits Course Questions, (URL: http://utwired.engr.utexas.edu/rgd1/lesson02.cfm

[23] Student study guide, Problem Solving, Egrade Assessment

[24] NSF-sponsored meeting on Concept Inventory (CI) Central,

, $\quad$ https://engineering.purdue.edu/SCI/workshop/index.html

[25] Electronics Concept Inventory

http://www.foundationcoalition.org/events/news/conferencepapers/2004asee/simoni.pdf

[26] Board for Professional Engineers Registration, http://www.pels.ca.gov/

[27] Reed Rhoads Commisssion Paper, http://www7.nationalacademies.org/bose/Reed_Rhoads_CommissionedPaper.pdf 